\title{
Thermal Analysis for Orbiter and ISS Plume Impingement on International Space Station
}

\author{
W. C. Rochelle, E. A. Reid, T. L. Carl, and R. N. Smith \\ Lockheed Martin Space Operations, Houston, TX \\ F. E. Lumpkin \\ NASA/Johnson Space Center, Houston, TX
}

\begin{abstract}
$\underline{\text { Abstract }}$
The NASA Reaction Control System (RCS) Plume Model (RPM) is an exhaust plume flow field and impingement heating code that has been updated and applied to components of the International Space Station (ISS). The objective of this study was to use this code to determine if plume environments from either Orbiter PRCS jets or ISS reboost and Attitude Control System (ACS) jets cause thermal issues on ISS component surfaces. This impingement analysis becomes increasingly important as the ISS is being assembled with its first permanent crew scheduled to arrive by the end of fall 2000 . By early summer 2001, the ISS will have a number of major components installed such as the Unity (Node 1), Destiny (Lab Module), Zarya (Functional Cargo Block), and Zvezda (Service Module) along with the P6 solar arrays and radiators and the Z-1 truss. Plume heating to these components has been analyzed with the RPM code as well as additional components for missions beyond Flight 6A such as the Propulsion Module (PM), Mobile Servicing System, Space Station Remote Manipulator System, Node 2, and the Cupola. For the past several years NASA/JSC has been developing the methodology to predict plume heating on ISS components. The RPM code is a modified source flow code with capabilities for scarfed nozzles and intersecting plumes that was developed for the 44 Orbiter RCS jets. This code has been validated by comparison with Shuttle Plume Impingement Flight Experiment (SPIFEX) heat flux and pressure data and with CFD and Method of Characteristics solutions. Previous analyses of plume heating predictions to the ISS using RPM have been reported, but did not consider thermal analysis for the components nor jet-firing histories as the Orbiter approaches the ISS docking ports. The RPM code has since been modified to analyze surface temperatures with a lumped mass approach and also uses jet-firing histories to produce pulsed heating rates. In addition, RPM was modified to include plume heating from ISS jets to ISS components where the jet coordinates are specified, together with the engine cant angle. These latter studies have been focused on the PM with plumes from its reboost and ACS jets impinging on various ISS components and also focused on the Japanese H2 Transfer Vehicle (HTV) with the plumes from its reboost engines impinging on the Cupola window. This paper will present plume heating and surface temperature results on a number of ISS components with and without jet-firing histories, evaluate post-flight data, and describe any potential thermal issues.
\end{abstract}

